\title{
Fragmentation of thin plates by dot impact
}

\author{
R.Ye.Brodskii, T.V.Kulik \\ Institute for Single Crystals, STC "Institute for Single Crystals" \\ National Academy of Sciences of Ukraine, \\ 60 Lenin Ave., 61001 Kharkiv, Ukraine
}

Received April 24, 2015

\begin{abstract}
In this paper we study the case of two-dimensional single-impact fragmentation, namely the fragmentation of the circular thin plate by dot impact. The distribution density of the fragments by mass was obtained analytically in the case of the central strike and numerically for arbitrary random point of impact. The study was conducted for different values of the number of fissures, the dependence of the distribution parameters on that number was obtained.
\end{abstract}

Keywords: fragmentation, thin round plates, dot impact.

\begin{abstract}
Исследован случай двумерной одноударной фрагментации, а именно фрагментация круглой тонкой пластины точечным ударом. Получена плотность распределения осколков по массе, аналитически в случае центрального удара и численно для произвольного случайного места удара. Исследование проведено для разных значений числа трещин, получена зависимость параметров распределения от этого числа.
\end{abstract}

Фрагментація тонких пластин точковим ударом. Р.Є.Бродсъкий, Т.В.Кулік.

Досліджено випадок двомірної одноударної фрагментації, а саме фрагментація круглої тонкої пластини точковим ударом. Отримано густину розподілу уламків за масою, аналітично у разі центрального удару i чисельно для довільного випадкового місця удару. Дослідження проведено для різних значень числа тріщин, отримано залежність параметрів розподілу від цього числа.

\section{Introduction}

Fragmentation or crushing of material one of the most widely used in engineering processes and one of the most common in nature [1]. Fragmentation processes are classified according to the characteristics of destructing objects and mode of action. In particular, one of the most common characteristics is the dimension of the destroyed object - one-, two- and three-dimensional material fragmentation [2]. An example of a one-dimensional fragmentation is cutting of long molecules or fragmentation rods, twodimensional fragmentation - the fragmentation of plates [2], three-dimensional volumetric bodies [3-10]. By the method of action one distinguishes the single-impact "weak" and many-impact "strong" (long- term) fragmentation [11]. In the case of single-impact fragmentation the result depends on initial shape of the sample and the method of striking, long-term fragmentation is often characterized by "forgetting" the initial conditions of the system [12].

In this paper we explore one of the cases of the two-dimensional fragmentation. The two-dimensional (or quasi-two-dimensional) fragmentation takes place, in particular, for any destruction of solid walls and, thus, study of the two-dimensional fragmentation is important from a practical point of view [13].

In the work we investigated the two-dimensional single-impact fragmentation. Namely, the fragmentation of plates under dot impact. The result of the destruction in such process depends on the plate shape, we studied the fragmentation of circular 


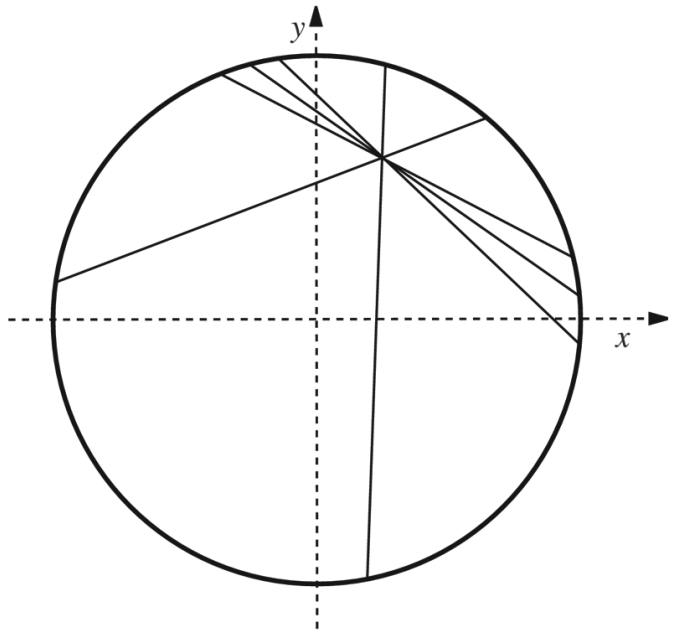

Fig. 1. Destruction of the circular plate by dot impact. The number of cracks on the figure $n=5$.

plates, this form is convenient for the study because of its symmetry.

In the paper we consider destruction of the circular plate of constant thickness by fissures, which form in the single dot strike. Cracks are direct, i.e. represent chords, intersect at the strike point. This point is randomly selected, its coordinates are uniformly distributed over the plate. We consider separately the case of strictly central impact, in this case an exact analytical solution was obtained.

On the plate it introduced the Cartesian coordinate system and the direction of fissure propagation is defined by the angle between the crack-chord and horizontal axis. This angle is uniformly distributed in the range $[0,2 \pi)$.

As a result, the plate is cut by fissures into many pieces. The aim of the work was to determine the distribution density of these fragments by mass. This density was found analytically for the case of the central strike and numerically for the arbitrary random position of the point of striking.

In expression for the density parameter, depending on the number of cracks is included. From a series of numerical experiments it was obtained an expression for this dependence.

\section{Problem statement}

Let's consider destruction of a circular plate of radius $R$ by dot impact. Let's introduce coordinate system with the origin at the center of the plate. Let the impact is made at the point with coordinates $(x, y)$. As a result of the impact the plate will split by $n$ fissures passing through the point $(x, y)$.

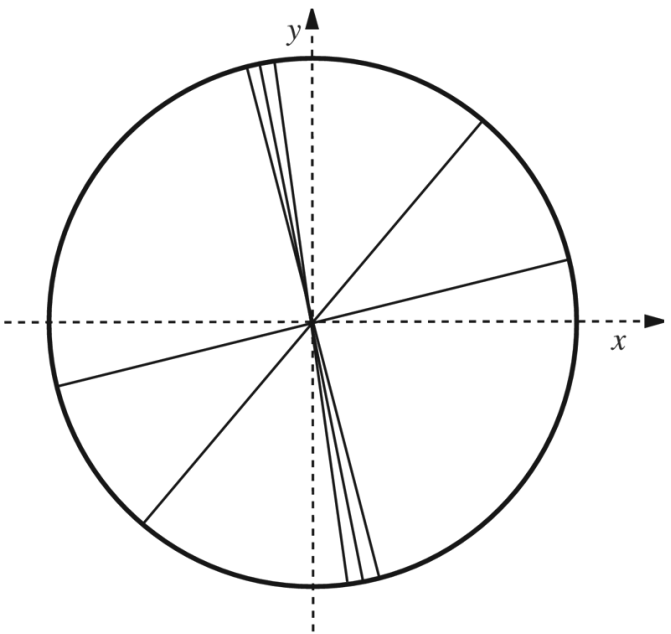

Fig. 2. Fragmentation of round plate by dot central impact.

We assume that the fissures lines, i.e. represent the chords (Fig. 1).

At the destruction $2 n$ fragments are produced (if two cracks are not the same).

In this paper we assume that the coordinates of the point of impact $(x, y)$ are random and uniformly distributed over the plate, the angle between the fracture and the horizontal are random and uniformly distributed in $[0,2 \pi)$.

The aim of work is to obtain the distribution of fragment masses. The fragment mass is obviously proportional to their area. For convenience, we assume that the surface density of the plate equal to $1 / \pi R^{2}$ thus, the mass of the fragment will be equal to the part of its area by the total area of the plate.

In this paper, the plate thickness and its density is considered as independent of the coordinates.

The number $n$ of the fissures we suppose to be greater than one, the case $n=1$ was considered in [14].

\section{The case of central impact}

In general, the expression for the fragments masses is rather cumbersome, so the solution should be sought in an approximate form (using different approximations for the "narrow" and "broad" fragments) or obtain the solution numerically. However, when the impact acts at the center (Fig. 2), it can obtain an exact solution for the mass distribution of the fragments explicitly.

Areas of the fragments in the case of the central impact are directly proportional to the angles $\alpha_{i}$ between the cracks, which form this fragment. One (any) fissure di- 
vides the plate into two halves, each of which is split up by the remaining $n-1$ fissures into the fragments-sectors. Thus, the problem of splitting the circular plate by $\mathrm{n}$ fissures by the central impact is analogous to the problem of cutting a segment of length $\pi$ by $n-1$ points on it.

Let's assume that the angle at which the crack goes evenly distributed in $[0,2 \pi)$. For $N$ points distributed uniformly over the unit interval the distribution density of lengths $l$ of segments between them is [15]

$$
p(l)=N(1-l)^{N-1} .
$$

In our case $N=n-1$, and interval is not unit, and it has "length" $\pi$. Thus, the distribution density of the angle $\alpha$ between two cracks is

$$
p(\alpha)=(n-1)(\pi-\alpha)^{n-2} / \pi^{n-1} .
$$

So the distribution density of fragments by mass $m=1 / \pi R^{2} \cdot \alpha R^{2} / 2=\alpha / 2 \pi$ is

$$
p(m)=(n-1) 2^{n-1(1 / 2-m)^{n-2}} .
$$

It can see that this expression has sense only when the number of cracks $n \geq 2$. Indeed, in the case of the central strike only one fissure will always divide the plate exactly in half. For $n=2$ distribution is uniform

$$
p(m)=2
$$

in interval $(0,1 / 2]$ of mass values. At $n>2$ distribution density has power form. Let's consider asymptotes. For the small masses $m<<1 / 2$ the distribution density will have the form

$$
p(m) \approx 2(n-1)(1-2(n-2) m),
$$

i.e., the density will be linear on $m$.

In the large-scale region $m \sim 1 / 2$ density behaves as power function on small $\delta=1 / 2-m$,

$$
p(m)=(n-1) 2^{n-1} \delta^{n-2}, \quad \delta=\delta(m) .
$$

\section{Case of arbitrary position of the impact point}

In the case of fragmentation of circular plate by strike at random position of the point of impact, the problem was solved by numerical simulation.

In the computer model a round plate with center at the origin was cut by $n$ chords passing through the selected point $(x, y)$. Coordinates $(x, y)$ were chosen randomly, uniformly

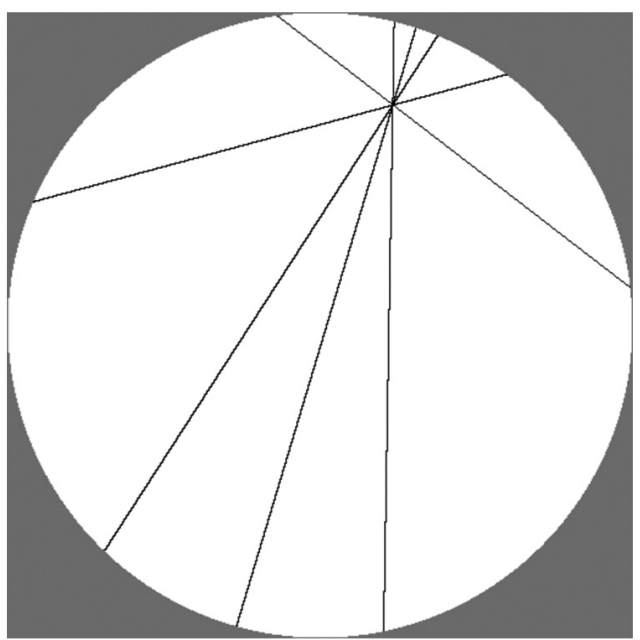

Fig. 3. Image of one of the simulated plates generated by simulation program.

on the plate surface. Angle $\alpha$ between the fissure and horizontal was also distributed randomly and uniformly in $[0,2 \pi$ ) (Fig. 3). The number $n$ of fractures was fixed, the experiment was carried out with the numbers $n=2 \ldots 10$. In the experiment the areas of fragments were calculated. 300 experiments were performed for each $n$.

Basing on the experiments results a histogram of distribution density of the fragments was constructed. Fig. 4 shows the histogram in logarithmic scale on the axis $y$ for $n=2, n=5, n=10$.

It can see that the histogram of the distribution density of the fragments in these coordinates is close to a straight line. Thus, a good approximation of the distribution density is an exponential dependence of the form

$$
p_{n}(m)=A_{n} \exp \left(-\gamma_{n} m\right), \quad \gamma_{n}>0 .
$$

Graphs of the exponential approximation also shown in Fig. 4. The coefficient $A_{n}-$ normalizing, density $p_{n}(m)$ is normalized to unity.

$$
\int_{0}^{1} p(m) d m=1,
$$

whence

$$
A_{n}=\gamma_{n} /\left(1-e^{\left.-\gamma_{n}\right)} .\right.
$$

The exponent $\gamma_{n}$ for $n=2 \ldots 10$ is respectively equal to

$n, \gamma_{n}$

$2,3.0001370$

$3,4.4445229$

$4,6.1403539$ 

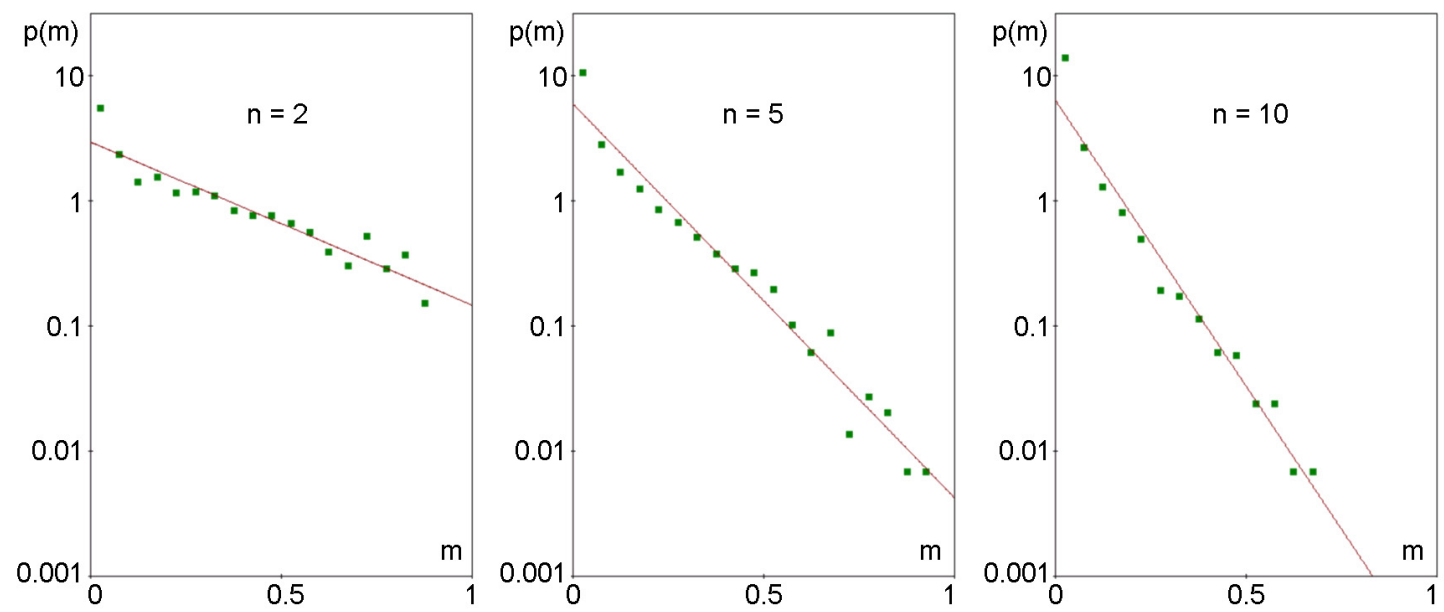

Fig. 4. Distribution density of the fragments by mass, numerical experiment for the number of fissures $n=2, n=5, n=10$.

$5,7.2494787$

$6,7.7284575$

$7,8.0978331$

$8,8.7964132$

$9,10.354894$

$10,10.533272$

Fig. 5 shows the values of $\gamma_{n}$ for $n=$ $2 \ldots 10$. In previous work [14] we analytically calculated the distribution density of the fragments in the case of the single crack under the same assumptions about distribution of the position of the impact point and angle $\alpha$ between the fracture and the horizontal. This density was constant

$$
p_{1}(m)=\text { const. }
$$

At the modeling program used above the numerical experiment for the case $n=1$ was also carried out. Distribution obtained in the experiment coincided with the found analytically that confirms the correctness of the simulation. We can supplement the values of $\gamma_{n}$, obtained above by value of $\gamma_{1}=0$. This point is also shown in Fig. 5 . Thus, the behavior $\gamma_{n}$ as function of $n$ should be sought in the form of the function for which $\gamma_{1}=0$. It was found that the experimentally determined values of the exponent $\gamma_{n}$ is very well approximated by the function of the form $\gamma_{n}=C \ln (n)$ (Fig. 5), where $C \approx 4,5$. Thus, the distribution density of the fragments can be written as

$$
p_{n}(m)=A_{n} n^{-C \cdot m}, \quad C=\text { const },
$$

where $A_{n}=\gamma_{n} /\left(1-\mathrm{e}^{\left.-\gamma_{n}\right)}=C \ln n /\left(1-n^{-C}\right)\right.$ at $n>1$. The corresponding probability distribution

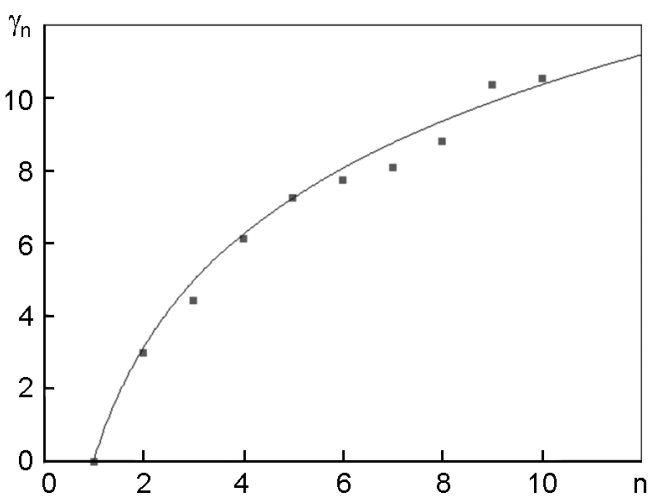

Fig. 5. Values of the exponent $\gamma_{n}$ of exponential approximation of distribution density of fragments by mass in dependence on $n, n=1 \ldots 10$. Dots show the experimental values, solid line - approximation $\gamma_{n}=C \ln (n)$.

$$
P_{n}(m)=\int_{0}^{m} p_{n}(m) d m=\frac{1-n^{-C \cdot m}}{1-n^{-C}} .
$$

Numerical experiment was also carried out for the case of the central impact, i.e. fixed coordinates $(x, y)=(0,0)$. Histogram for this case is shown in Fig. 6. Also in Fig. 6 it is shown the distribution density, found analytically above. It can be seen that the distribution resulting in the numerical simulation agrees very well with the expression obtained analytically, that confirms the correctness of the simulation.

\section{Conclusions}

Thus, in this paper we obtain distribution density of masses of the fragments at the fragmentation of circular thin plate by single dot impact. 


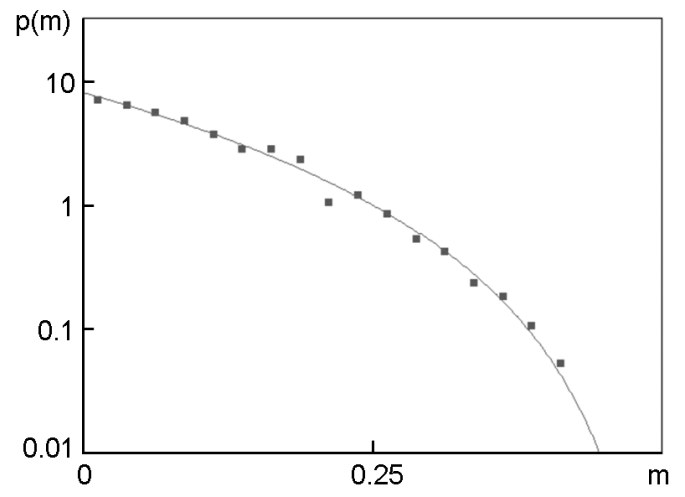

Fig. 6. Distribution density of the fragments by masses in the case of the center impact.

In the case of the central strike the expression for the density was obtained analytically and had the form

$$
p(m)=(n-1) 2^{n-1}\left(\frac{1}{2}-m\right)^{n-2}, m \in\left[0, \frac{1}{2}\right],
$$

where $n$ - number of cracks. Density has a linear asymptotic behaviour at the low masses and the power in the large-scale area.

In the case of arbitrary random position of the point of impact the density was obtained numerically. Good approximation of the data is the exponential function

$$
p_{n}(m)=A_{n} \exp \left(-\gamma_{n} m\right), \gamma_{n}>0, m \in[0,1],
$$

were $\gamma_{n}=C \cdot \ln n$.

This result is consistent with the expression obtained in [14] for the distribution density of the fragments in fragmentation of the plate by one fissure, i.e, for $n=1$. This density has the form $p_{1}(m)=$ const.

With the substitution $\gamma_{n} \approx C \ln (n), C \approx 4.5$ obtained from the numerical experiment, and the normalization factor $A_{n}=C \ln n /\left(1-n^{-C}\right)$ the distribution density at $n>1$ is equal to

$$
p_{n}(m)=C \frac{\ln n}{1-n^{-C}} n^{-C \cdot m} .
$$

The probability distribution thus equals

$$
P_{n}(m)=\frac{1-n^{-C \cdot m}}{1-n^{-C}} .
$$

The numerical experiment was carried out for the case of the central strike. The results fully consistent with the analytical solution, that confirms the correctness of the simulation.

\section{References}

1. V.V.Yanovsky, A.V.Tur, O.V.Kuklina, JETP, 137, 1 (2010).

2. P.L.Krapivsky, E.Ben-Naim, Phys.Rev.E, 50, 3502 (1994).

3. D.L.Turcotte, J.Geophys.Res., 91, 1921 (1986).

4. B.R.Lawn, T.R.Wilshaw, Fracture of Brittle Solids, Cambridge University Press, Cambridge (1975).

5. R.Shinnar, J.Fluid Mech., 10, 259 (1961).

6. K.C.Chase, P.Bhattacharyya, A.Z.Mekjian, Phys.Rev. C, 57, 822 (1998).

7. S.Redner, Statistical Models for the Fracture of Disordered Media, ed. by H.J.Herrmann, S.Roux, Elsevier Science, New York (1990).

8. T.Ishii, M.Matsushita, J.Phys.Soc.Jap., 61, 3474 (1992).

9. L.Oddershede, P.Dimon, J.Bohr, Phys.Rev. Lett., 71, 3107 (1993).

10. T.Kadono, Phys.Rev. Lett., 78, 1444 (1997).

11. O.V.Kuklina, V.V.Mozgin, A.V.Tur, V.V.Yanovsky, Functional Materials, 8, 233 (2001).

12. Yu.P.Virchenko, R.Ye.Brodskii, Modern Probl. Natur. Scie., 1(2), 81 (2014).

13. R.Ye.Brodskii, P.V.Konevsky, R.I.Safronov, Functional Materials, 18, 200 (2011).

14. R.Brodskii, A.V.Tur, V.V.Yanovsky, Functional Materials, 19, 525 (2012).

15. M.Kendall, P.Moran, Geometric Probabilities, Nauka, Moscow (1972) [in Russian]. 